\title{
Socio-Cultural Factors Associated with Antenatal Services Utilization: A Cross- Sectional Study in Eastern Nepal
}

Krishna Kumar Deo ${ }^{1}$ and Ravi Kumar Bhaskar ${ }^{*}$

${ }^{1}$ Institute of Medicine, Kathmandu, Nepal

${ }^{2}$ Department of Community Medicine and Public Health, National Medical College, Birgunj, Nepal

*Corresponding author: Ravi Kumar Bhaskar, Department of Community Medicine and Public Health, National Medical College, Birgunj, Nepal, Tel: 00977-9804363736; E-mail: hi.rkvaskar@gmail.com

Received date: Feb 07, 2014, Accepted date: Dec 09, 2014, Published date: Dec 16, 2014

Copyright: ( $) 2014$ Bhaskar RK, et al. This is an open-access article distributed under the terms of the Creative Commons Attribution License, which permits unrestricted use, distribution, and reproduction in any medium, provided the original author and source are credited.

\section{Abstract}

Background: This study was done to assess the socio-economic factors associated with Antenatal care (ANC) service utilization in Eastern Nepal.

Methods: A cross-sectional quantitative study was conducted in Sunsari district of Eastern Nepal. A total of 372 Women delivered within period of one year preceding and this study were interviewed through household visit.

Results: Logistic regression analyses revealed that women exposed to media had more than three times higher chance of receiving four or more ANC services (AOR=3.48, 95\%Cl:1.20-10.05). Similarly, women from advantaged ethnicity had more than two times higher chance of having four or more ANC visits than respondents who were from disadvantaged ethnicity $(\mathrm{AOR}=2.43,95 \% \mathrm{Cl}$ : 2.04-6.92). Similarly, women having higher level of autonomy were nearly three times more likely to have four or more ANC visits than the women having lower level of autonomy $(A O R=2.86,95 \% \mathrm{Cl}: 1.47-5.64))$.Similarly richer women were two times more likely to have four or more ANC visits than poorer women $(\mathrm{AOR}=2.28,95 \% \mathrm{Cl}$ : 1.06-5.25).

Conclusion: Relatively advantaged ethnicity, higher rank of women's autonomy, good knowledge on maternal health service and knowledge on incentive upon completion of ANC, media exposure with maternal health service related massages and higher wealth rank were found significantly associated with 4 time ANC service utilization.

Keywords: Socio-cultural; Factors; Antenatal; Maternal; Health; Services; Nepal

\section{Introduction}

Despite remarkable progress in last fifteen years, Nepal has still high maternal mortality ratio (MMR) compared to many developed countries [1]. The MMR in Nepal was estimated to be 229 per live 100000 births. In Nepal, maternal deaths account for 11 percent of all deaths among women of reproductive Age, 15-49 years [2].

Most maternal deaths and pregnancy related complications can be prevented if pregnant women have access to good quality maternal health services which includes services during pregnancy (antenatal care), during delivery (intra-natal care) and after delivery (postnatal care). Antenatal care (ANC) is care during pregnancy which includes monitoring blood pressure, weight and fetal heart rate; Providing information, education and communication (IEC) and behavior change communication (BCC) for danger signs and care during pregnancy; timely referral to the appropriate health facilities; Birth preparedness and complication readiness (BPCR) for both normal and obstetric emergencies; Early detection and management of complications; Provision of tetanus toxoid (TT) immunization, iron tablets, deworming tablets to all pregnant women and malaria prophylaxis where necessary [3]. ANC is also essential for diagnosing and treating complications. ANC is a potentially important way to connect a woman with the health system. Care during pregnancy is an important opportunity to deliver interventions that will improve maternal health and survival during the period immediately preceding and after birth.

ANC offers an important opportunity for healthcare providers to inform women about the advantages of delivering their babies with the help of an SBA. Moreover, ANC teaches pregnant women about the danger signs of pregnancy, making them capable to recognize early symptoms and go to a health facility as soon as possible. For women with normal pregnancies, WHO recommends a minimum of four ANC visits, ideally at $16,24-28,32$, and 36 weeks [4].

Ministry of Health and Population, Nepal also recommends a minimum of four ANC visits which is provided from the network of its health system extended up to the community level. District Hospitals, primary health care centers, health/sub-health posts and primary health care outreach clinics are the key service providing institutions at district and peripheral level in Nepalese health care system [5].

To increase demand for and improve access to maternity services, Government of Nepal launched Maternity Incentives scheme in 2005 and provide each woman a fixed amount of money to cover transportation costs, based on ecological region. Thus, women in the mountainous, hilly, and plain Tarai regions receive 1,500,1,000, and 500 Nepalese rupees (Rs.) (1 US\$ $\approx$ Rs. 96 in August 2013), respectively, for each birth if she delivers in government health institutions. Apart from this, a woman receives additional incentives (Rs.400) if she 
completes four ANC visits, institutional delivery and first PNC. The government has also implemented the Aama Suraksha (Mothers' Safety) Programme and birth preparedness package (BPP) in all 75 districts to encourage institutional delivery. This programme combines free delivery services at any public health facility and a number of private facilities with the maternity Incentives schemes $[4,6]$.

In spite of government's intensive endeavors, there are wide disparities in maternal health service utilization among women residing in rural part and belonging to lowest wealth quintiles [7]. Along with strengthening the maternal health service, socio cultural influences in seeking health services, economic and physical accessibility of mothers to the health facility are the crucial factors that must be taken into consideration to improve the maternal service utilization [2].

\section{Materials and Methods}

A cross-sectional quantitative study was conducted in three VDCs of Sunsari district in Eastern Nepal. The VDCs were selected randomly. Women who had delivered within period of one year preceding this study and who gave informed consent were interviewed.

Sample size was calculated by using the formula (33):

$\mathrm{N}=1.96^{2} \mathrm{PQ} / \mathrm{L}^{2}$

P is the prevalence of institutional delivery from NDHS 2011, Q is the complement of $P$, i.e.,

$\mathrm{Q}=100-\mathrm{P}, \mathrm{L}$ is precision/allowable error, which is taken to be $5 \%$ in this study. $=\left(1.96^{\star} 1.96^{\star} 35.5^{\star} 64.5\right) / 5^{\star} 5$

$$
=351.85 \ldots . .352 \text { (approx) }
$$

Thus, amplifying by $5 \%$ for possible non-response, the final sample size is $352+18=370$.

Before the mothers were approached through sub health posts, a written permission was taken from District Health Office (DHO), Sunsari to assist in survey at community level. Sub health posts are government run health institutions which are under DHO. SHPs are the main health service providing institutions with in the VDCs. The SHP in-charges were consulted to get updated list of children born and immunized during last one year period. A list of children who were immunized with in defined one year period preceding the study was prepared from immunization register maintained by VHWs of respective VDCs. A complete list of children was expected from immunization register as the VDCs had more than 100 percent BCG coverage in the recent years. The exhaustive list of 1064 household heads having less than one year children (children born during the period -5th June 2011 to 6th July 2012) was prepared from the immunization register from the three VDCs. A total of 1064 children born during the period and immunized in the three VDCs. List of the parents (head of household) was prepared to trace the household with mothers having under one year children. A total of 372 households out of 1064 were identified through systematic random sampling method. From these households, Women who delivered within period of one year preceding this study were interviewed through household visit.

In case of non-response, plan was made to move to another immediate mother in the list. A total of 16 mothers could not meet during household visit. Subsequent mothers in the list were interviewed instead of them. Structured questionnaire was originally prepared in English and later translated into local language, Maithili and was used to collect the quantitative data. The questionnaire was prepared through reviewing available literatures and focusing on objectives of the study. Data checking, editing and coding was done by the researcher each day. Data entry was done in Epi Data version 3 and analysis was done in SPSS 17. Data coding, recording and cleaning was continuously carried out to ensure data quality.

Descriptive analysis and bivariate analysis of service utilization and associated factors was done as per the study variables at the initial stage. Chi-square test was applied to test whether the factors were significantly associated ( $\mathrm{p}$ value $<0.05$ ) with service utilization at $95 \%$ confidence interval $(95 \% \mathrm{CI})$. Odds ratio (OR) with $95 \% \mathrm{CI}$ was also calculated to quantify the associated risk factors. The significant variables at $90 \% \mathrm{CI}(\mathrm{p}$-value $<0.1)$ observed in bivariate analysis was subjected to multivariate analysis (logistic regression). Adjusted odds ratio was calculated to measure the net effect size of variables. Hosmer and Lameshow Chi- square test was used to test the goodness of fit for regression model. A finding of non significance with $p$ values $(p>0.05)$ was concluded that the model adequately fits the data.

Ethical approval was taken from the Institutional Ethical Review Board, Institute of Medicine (IOM). Informed consent was taken from each respondent. Confidentiality and anonymity were assured and maintained.

\section{Result}

A total of 372 mothers were interviewed, of which 257 (69.1\%) women received 4 or more ANC visits while 49 (13.2\%) women received no ANC visits. More than six percent of respondent were below 20 years old. There was 44 percent of the respondents from 20-24 years age group and 27 percent from age group 25-30 years while 22 percent were of 30 years or more. More than three quarter women $(77.2 \%)$ were hindus while rest were Muslims. Most of the mothers were multiparous $(72.3 \%)$ while 27 percent were primiparous. Almost 50 percent of the mothers were from nuclear family. Nearly half $(48.1 \%)$ of the respondents were from disadvantaged ethnic group and most of them (73.9\%) were farmers. More than one third $(38.2 \%)$ of the respondents didn't go to school in their life and only $4.6 \%$ of the respondents were secondary school or upper level passed. Nearly two third $(62.4 \%)$ of respondents expressed their belief in traditional healer. About 45.7 percent of respondents had exposed to media depicting message related to maternal health service while about 76 percent had exposure with such media for at least once a week. The findings showed that about 60 percent mothers had information of free delivery service while about 69 percent mothers had information on transportation incentive on utilization of institutional delivery service. Among the interviewed mothers, almost 66 percent of mothers were in contact with FCHV during last delivery. About 32 percent of mothers had experienced complications during last or previous pregnancies and deliveries. Out of mothers being in contact with FCHV, 54 percent had met for two or more time during last pregnancy. The main reasons shared by mothers for utilizing ANC service was for better health of mother and baby (49.4\%) and for complication assessment (32.1\%). About 32.5 percent of mothers shared that they were advised by FCHV for the utilization of ANC services.

Table 1 showed that mothers from relatively advantaged ethnicity were more likely to receive 4 or more ANC visit than disadvantaged 
Citation: Deo KK, Bhaskar RK (2014) Socio-Cultural Factors Associated with Antenatal Services Utilization: A Cross-Sectional Study in Eastern

Page 3 of 6

(Dalit and Muslim) ethnic group (OR=4.62, 95\%CI: 2.84-7.49). Religion was significantly associated with ANC service utilization. Hindu mothers were more likely to receive ANC services than Muslims (or=3.73, 95\%CI: 2.25-6.18). Mothers having High (very good) knowledge regarding maternal health services were more likely to receive ANC services (OR=18.40, 95\%CI: 8.71- 41.03) than mothers having poor knowledge, as shown in Table 2.

\begin{tabular}{|c|c|c|c|c|}
\hline \multirow{2}{*}{ Characteristics } & $<4$ ANC & $\geq 4$ ANC & \multirow{2}{*}{$P$ Value } & \multirow{2}{*}{ OR $(95 \% \mathrm{Cl})$} \\
\hline & $n=115$ & $\mathrm{n}=\mathbf{2 5 7}$ & & \\
\hline \multicolumn{5}{|l|}{ Ethnicity } \\
\hline Disadvantaged & 84 & 95 & & \\
\hline Relatively advantaged & 31 & 162 & 0.001 & $4.62(2.84-7.49)$ \\
\hline \multicolumn{5}{|l|}{ Religion } \\
\hline Hindu & 69 & 218 & & \\
\hline Muslim & 46 & 39 & 0.001 & $0.26(0.16-0.44)$ \\
\hline \multicolumn{5}{|l|}{ Education of respondent } \\
\hline Illiterate & 10 & 13 & & \\
\hline Literate & 105 & 244 & 0.178 & $1.78(0.76-4.26)$ \\
\hline \multicolumn{5}{|l|}{ Education of husband } \\
\hline Illiterate & 19 & 19 & & \\
\hline Literate & 96 & 238 & 0.007 & $2.47(1.28-4.87)$ \\
\hline \multicolumn{5}{|c|}{ Occupation of respondent } \\
\hline Service/Business & 15 & 32 & & \\
\hline Agriculture/Wage labour & 100 & 225 & 0.874 & $1.05(0.54-2.04)$ \\
\hline \multicolumn{5}{|l|}{ Occupation of husband } \\
\hline Service/Business & 48 & 113 & & \\
\hline Agriculture/Wage Labour & 67 & 144 & 0.88 & $0.91(0.63-1.44)$ \\
\hline \multicolumn{5}{|c|}{ Belief in traditional healer's practices } \\
\hline No & 16 & 124 & & \\
\hline Yes & 99 & 133 & 0.001 & $0.17(0.09-0.31)$ \\
\hline \multicolumn{5}{|l|}{ Wealth quintile } \\
\hline First(Poor) & 37 & 37 & & \\
\hline Second & 29 & 46 & 0.165 & $1.58(0.82-3.41)$ \\
\hline Third & 20 & 49 & 0.011 & $2.45(1.22-4.89)$ \\
\hline Fourth & 18 & 62 & 0.001 & $3.44(1.72-6.57)$ \\
\hline Fifth(Rich) & 11 & 63 & 0.001 & $5.72(2.65-2.36)$ \\
\hline \multicolumn{5}{|l|}{ Women's autonomy } \\
\hline Poor & 57 & 68 & & \\
\hline Medium & 38 & 94 & 0.006 & $2.07(1.23-3.47)$ \\
\hline Higher & 20 & 95 & 0.001 & $3.19(2.11-7.23)$ \\
\hline
\end{tabular}

Table 1: Association of socio cultural and economic factors with utilization of 4 or more ANC. 
Citation: Deo KK, Bhaskar RK (2014) Socio-Cultural Factors Associated with Antenatal Services Utilization: A Cross-Sectional Study in Eastern

\begin{tabular}{|c|c|c|c|c|}
\hline \multirow{2}{*}{ Characteristics } & $<4$ ANC & $\geq 4$ ANC & \multirow{2}{*}{$P$ Value } & \multirow{2}{*}{ OR $(95 \% \mathrm{Cl})$} \\
\hline & $n=115$ & $\mathrm{n}=\mathbf{2 5 7}$ & & \\
\hline \multicolumn{5}{|l|}{ Media exposure } \\
\hline No & 81 & 89 & & \\
\hline Yes & 34 & 168 & 0.001 & $4.17(2.78-7.77)$ \\
\hline \multicolumn{5}{|c|}{ Frequency of media exposure } \\
\hline At least once a week & 38 & 147 & & \\
\hline Less than once a week & 25 & 34 & 0.001 & $0.35(0.18-0.65)$ \\
\hline \multicolumn{5}{|l|}{ Contact with FCHV } \\
\hline No & 59 & 65 & & \\
\hline Yes & 56 & 192 & 0.001 & $3.11(1.75-4.78)$ \\
\hline \multicolumn{5}{|l|}{ Frequency of contact } \\
\hline One time & 43 & 93 & & \\
\hline Two or more time & 13 & 101 & 0 & $3.59(1.88-7.15)$ \\
\hline \multicolumn{5}{|c|}{ Pregnancy/Obstetric complication } \\
\hline No & 76 & 157 & & \\
\hline Yes & 39 & 100 & 0.357 & $1.27(0.83-1.94)$ \\
\hline \multicolumn{5}{|l|}{ Sex of previous child } \\
\hline Male & 49 & 96 & & \\
\hline Female & 50 & 98 & 0.991 & $1.00(0.61-1.62)$ \\
\hline \multicolumn{5}{|c|}{ Knowledge on maternal health services } \\
\hline Poor & 62 & 47 & & \\
\hline Medium & 44 & 81 & 0.001 & $2.42(1.43-4.11)$ \\
\hline High & 9 & 129 & 0.001 & $18.40(8.71-41.03)$ \\
\hline \multicolumn{5}{|c|}{ Knowledge on transportation incentive } \\
\hline No & 99 & 109 & & \\
\hline Yes & 16 & 148 & 0 & $8.40(4.66-15.08)$ \\
\hline
\end{tabular}

Table 2: Association of perceived need/benefit related factors with utilization of ANC services.

Table 3 shows Logistic regression analyses which revealed that women exposed to media were more than three times higher chance of receiving four or more ANC services (AOR=3.48, 95\%CI: 1.20-10.05). Similarly, women from advantaged ethnicity had more than two times higher chance of having four or more ANC visits than respondents who were from disadvantaged ethnicity (AOR=2.43, 95\%CI: 2.04-6.92).

Similarly, women having higher level of autonomy were nearly three times more likely to have four or more ANC visits than the women having lower level of autonomy $(\mathrm{AOR}=2.86,95 \% \mathrm{CI}$ : 1.47-5.64)). Similarly richer women were two times more likely to have four or more ANC visits than poorer women $(\mathrm{AOR}=2.28,95 \% \mathrm{CI}$ : 1.06-5.25). Similarly, women with good knowledge on maternal health services were more likely to have four or more ANC visits than the women having poor knowledge $\mathrm{AOR}=5.44,95 \% \mathrm{CI}$ : 1.26-23.33).

\section{Discussion}

This study showed a moderate prevalence (69.1\%) of ANC utilization for four or more times. This was higher than other recent national study $[4,7]$, which might indicate some progress. 
Citation: Deo KK, Bhaskar RK (2014) Socio-Cultural Factors Associated with Antenatal Services Utilization: A Cross-Sectional Study in Eastern

\begin{tabular}{|c|c|c|c|c|}
\hline \multirow{2}{*}{ Characteristics } & $<4$ ANC & $\geq 4$ ANC & \multirow{2}{*}{ Crude OR( $95 \% \mathrm{Cl})$} & \multirow{2}{*}{ Adjusted OR $(95 \% \mathrm{Cl})$} \\
\hline & $n=115$ & $\mathrm{n}=\mathbf{2 5 7}$ & & \\
\hline \multicolumn{5}{|l|}{ Media exposure } \\
\hline No & 81 & 89 & & \\
\hline Yes & 34 & 168 & $4.17(2.72-7.77)$ & $3.48(1.20-10.05)$ \\
\hline \multicolumn{5}{|c|}{ Frequency of media exposure } \\
\hline At least once a week & 38 & 147 & & \\
\hline Less than once a week & 25 & 34 & $0.35(0.18-0.65)$ & $0.75(0.26-2.16)$ \\
\hline \multicolumn{5}{|l|}{ Contact with FCHV } \\
\hline No & 59 & 65 & & . \\
\hline Yes & 56 & 192 & $3.11(1.75-4.72)$ & $0.72(0.51-2.14)$ \\
\hline \multicolumn{5}{|c|}{ Knowledge on maternal health services } \\
\hline Poor & 62 & 47 & & \\
\hline High & 58 & 189 & $18.4(8.17-41.3)$ & $5.44(1.26-23.33)$ \\
\hline \multicolumn{5}{|c|}{ Knowledge on incentive upon completion of 4 ANC } \\
\hline No & 99 & 109 & & \\
\hline Yes & 16 & 148 & $8.40(4.66-15.8)$ & $4.85(1.45-16.14)$ \\
\hline \multicolumn{5}{|c|}{ Belief in traditional healer's practices } \\
\hline No & 16 & 124 & & \\
\hline Yes & 99 & 133 & $0.17(.09-.30)$ & $0.05(0.01-0.26)$ \\
\hline \multicolumn{5}{|l|}{ Ethnicity } \\
\hline Disadvantaged & 84 & 95 & & \\
\hline Relatively advantage & 31 & 162 & $4.62(2.84-7.44)$ & $2.43(2.04-6.92)$ \\
\hline \multicolumn{5}{|l|}{ Women's Autonomy } \\
\hline Low & 57 & 68 & & \\
\hline High & 58 & 189 & $3.12(2.18-7.2)$ & $2.86(1.47-5.64)$ \\
\hline \multicolumn{5}{|l|}{ Wealth rank } \\
\hline Low(Poor) & 37 & 37 & & \\
\hline High(Rich) & 78 & 220 & $2.45(1.27-4.8)$ & $2.28(1.06-5.25)$ \\
\hline
\end{tabular}

Table 3: Multivariate analysis of socio cultural and perceived benefit/need related factors associated with ANC service utilizations.

The current study confirmed an association between knowledge about maternal health services and ANC utilization (i.e., mothers having good knowledge about maternal health services were more than 5 times more likely to use ANC services compared to those who had poor knowledge). Similarly, mothers having knowledge on incentive upon completion of 4 ANC visit were more likely (nearly 5 times) to use 4 ANC services than the mothers without such knowledge. Mothers exposed to media were nearly three and half times more likely to complete 4 ANC visit than the mothers not exposed to media. In the current study, exposure to media with maternal health service related message was found significantly linked with ANC practice similar to the study in Pakistan and Kenya that showed the association of media exposure with service utilization $[8,9]$. Belief in traditional healer, practice was also found significantly associated with maternal health service utilization which was similar to results from a study in Bangladesh [10]. Although bivariate analyses in this study showed positive association between education and ANC utilization, multivariate analyses in this study did not show significant association with education level of respondents with utilization of 
ANC services which was Contrary to the findings of many studies, [11-14].

In our study, women from relatively advantaged ethnicity used ANC services more frequently than women from disadvantaged ethnicity. Women from relatively advantaged ethnicity were approximately two and half times more likely to utilize 4 ANC visit than women from disadvantaged ethnicity. In this study Muslim and Dalit mothers are categorized under disadvantaged group.

The results of bivarite analysis also showed that Muslim mothers were less likely to utilize four times ANC service. This finding is similar to a study done in Nepal [15], however, contrary to another study in Nepal [11]. Higher status of women's autonomy was found significantly associated with four times ANC service utilization. This was similar to findings from the studies of Pakistan [16] and Nepal [17]. Women's autonomy is linked with decision making power of women to utilize health services.

In our study, wealth rank was found associated with 4 ANC visit. Rich women were two times more likely to complete 4 ANC visit than poor women. Economic status is linked with access to many services. Moreover, health service is generally linked with cost. Economic status depicted by wealth rank was significantly associated with 4 ANC visit. Various studies have tried to depict economic status by various ways (assets possession, amenity scoring, and income) which have produced difficulty in comparison. However, all the research showed that higher economic status is linked with higher service utilization $[12,16,18,19]$.

The factors associated with ANC utilization were ethnicity, women's autonomy, women's wealth rank, media exposure, good knowledge on maternal health services, knowledge on incentive upon completion of $4 \mathrm{ANC}$, and belief in traditional healer practices.

\section{Conclusion}

Relatively advantaged ethnicity, higher rank of women's autonomy, good knowledge on maternal health service and knowledge on incentive upon completion of ANC, media exposure with maternal health service related massages and higher wealth rank were found significantly associated with 4 time ANC service utilization. Education, occupation, age, parity, complication experienced during previous pregnancies, sex of previous child were not found significantly associated with 4 time ANC service utilization.

\section{Acknowledgements}

We would like to express our gratitude to the respondents who gave us their valuable time for completion of this study.

\section{References}

1. World Health Organization. Trends in Maternal Mortality: 1990-2010. Estimates developed by WHO, UNICEF, UNFPA and the World Bank. Geneva.
2. Pradhan A, Subedi BK, Barnett S (2010) Nepal Maternal Mortality and Morbidity Study 2008/2009.

3. Annual Report 2011/2012, Kathamandu: Department of Health Service, Ministry of Health and Population, GoN, Kathmandu, Nepal.

4. Choulagai B, Onta S, Subedi N, Mehata S, Bhandari GP, et al. (2013) Barriers to using skilled birth attendants' services in mid- and far-western Nepal: a cross-sectional study. BMC International Health and Human Rights13:49.

5. Paudel M, Khanal V, Acharya B, Adhikari M (2013) Determinants of Postnatal Service utilization in a Western District of Nepal: Community Based Cross Sectional Study. J Women's Health Care 2: 126.

6. (2005) Ministry of Health and Population (MoHP) Nepal, Ama Surkshya Program Implementation Guideline.

7. (2011) Ministry of Health and Population (MoHP) Nepal, New ERA, and Marco International Inc. Nepal Demographic and Health Survey (NDHS).

8. Agha S, Carton WT (2011) Determinant of Institutional Delivery in Rural Jhang, Pakistan. International Journal for Equity in Health10: 31.

9. Eijk AM, Bles HM, Odhiambo F, Ayisi JG, Blokland IE (2006) Use of ANC Services and Delivery Care among Women in Rural Kenya: A Community Based Survey. Reproductive Health 3: 2 .

10. Chakraborty N, Islam MA, Chowdhury RI, Islam R, Bari W, et al. (2003) Determinants of the Use of Maternal Health Services in Rural Bangladesh. Health Promotional International 18: 327-337.

11. Baral YR, Lyons K, Skinner J, ER, Teijlingen V (2010) Determinants of Skilled Birth Attendants for Delivery in Nepal. Kathmandu University Medical Journal 8: 31.

12. Letamo G, Rakgasi SD (2003) Factors Associated with Non Use of Maternal Health Services in Botswana. J Health popoulation Netr 21: 40-47.

13. Sathar ZA, Shahnaz K (2010) Women's Autonomy in the Context of Rural Pakistan. The Pakistan Development Review 39: 89-110.

14. Mall DS, Giri K, Karki C, Chaudhary P (2011) Achieving Millennium Development Goals 4 and 5 in Nepal. BJOG An international Journal of Obstetrics 8: 31 .

15. Shaky S (2006) Factors Influencing Utilization of Maternal and Neonatal Health Services among Ethnic Group in Nepal. Population and Reproductive Health Research 9: 15.

16. Sathar ZA, Shahnaz K (2010) Women's Autonomy in the Context of Rural Pakistan. The Pakistan Development Review 39: 89-110.

17. Acharya DR (2010) Women's Autonomy in Household Decision Making: A Demographic Study in Nepal. Reproductive Health 7: 15.

18. Wagle RR, Sabroe S, Nielsen BB (2004) Socioeconomic and physical distance to the maternity hospital as predictors for place of delivery: an observation study from Nepal. BMC pregnancy and Childbirth 4: 8.

19. Rashid SF, Hadi A, Afsana K, Begum SA (2001) Acute Respiratory Infections in Rural Bangladesh: Cultural Understandings, Practices and Role of Mothers and Community Health Volunteers. Tropical Medicine and International Health 6: 249-255. 